\title{
TREINAMENTO FÍSICO NO RISCO DE DOENÇA ISQUÊMICA CARDÍACA EM SUJEITOS HIV/AIDS EM USO DE TARV
}

\author{
PHYSICAL TRAINING ON THE RISK OF ISCHEMIC HEART DISEASE IN HIV/AIDS \\ SUBJECTS ON HAART \\ ENTRENAMIENTO FÍSICO CON RIESGO DE CARDIOPATÍA ISQUÉMICA EN SUJETOS \\ CON VIH/SIDA TRATADOS CON HAART
}

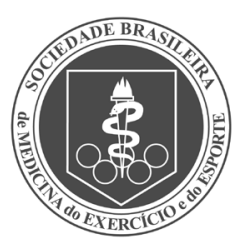

Artigo Original

\author{
Alexandre Ramos Lazzarotto \\ (Educador Físico) \\ Fernanda Bissigo Pereira² \\ (Nutricionista) \\ Angela d'Avila Harthmann ${ }^{3}$ \\ (Educador Físico) \\ Karen Olivia Bazzo² (Biomédica) \\ Fábio Longo Vicenzi ${ }^{4}$ (Biomédico) \\ Eduardo Sprinz 5 (Médico) \\ 1. Unilasalle, Canoas, RS, Brasil. \\ 2. Faculdade da Serra Gaúcha \\ (FSG), Caxias do Sul, RS, Brasil. \\ 3. Centro Universitário Metodista \\ (IPA), Porto Alegre, RS, Brasil. \\ 4. Universidade Feevale, Porto \\ Alegre, RS, Brasil. \\ 5. Universidade Federal do Rio \\ Grande do Sul (UFRGS), Porto \\ Alegre, RS, Brasil.
}

\section{Correspondência:}

Av. Vitor Barreto, 2288, Canoas, RS, Brasil. 92010-000.

alazzar@terra.com.br

\section{RESUMO}

Introdução: A terapia antirretroviral combinada (TARV) foi introduzida no Brasil em 1996, como parte da política nacional de acesso gratuito aos serviços de saúde e medicamentos. Infelizmente, o seu uso contínuo tem sido associado com mudanças na distribuição da gordura corporal e com alterações metabólicas que podem aumentar a morbidade e mortalidade nesta população. O treinamento físico tem sido estudado como uma estratégia eficaz de intervenção não farmacológica para melhorar os parâmetros de aptidão física relacionados à saúde e para minimizar os efeitos indesejáveis da infecção pelo HIV e/ou o uso prolongado da TARV, no entanto, há poucos estudos sobre o treinamento físico, síndrome lipodistrófica e cardiologia. Objetivo: Avaliar o risco de doença isquêmica cardíaca em sujeitos HIV/AIDS em uso de TARV praticantes de treinamento concorrente com séries simples. Métodos: Quatorze sujeitos foram avaliados através da circunferência abdominal, pressão arterial sistólica (PAS) e diastólica (PAD), colesterol total (CT), HDL, LDL, triglicerídeos (TG) e glicemia. Para a estimativa do risco coronariano em 10 anos utilizou-se o Escore de Framingham. Resultados: A maioria dos sujeitos situou-se dentro dos valores de referência para as variáveis analisadas, exceto para os valores de LDL e TG. Treze sujeitos (92,7\%) ficaram abaixo dos $10 \%$ de risco coronariano em 10 anos, e apenas um (7,3\%) estava em risco moderado. Houve correlação significativa entre o tempo de treinamento e a PAS. Conclusão: Sugere-se a realização de ensaios clínicos randomizados para avaliar os mesmos desfechos deste estudo.

Palavras-chave: exercício, coronariopatia, HIV/AIDS.

\begin{abstract}
Introduction: Highly active antiretroviral therapy (HAART) was introduced to Brazil in 1996, as part of the national policy of free access to health services and drugs. Unfortunately, its continued use has been associated with changes in the redistribution of body fat, and metabolic changes which can increase morbidity and mortality in this population. Physical training has been studied as an effective strategy of non-pharmacological intervention to improve physical fitness parameters related to health and to minimize the undesirable effects of HIV infection and/or prolonged use of HAART. However, there are few studies on physical training, lipodystrophy syndrome and cardiology. Objective: To assess the risk of ischemic heart disease in HIV/AIDS subjects on HAART and in concurrent training with single sets. Methods: Fourteen subjects were assessed by waist circumference, systolic (SBP) and diastolic (DBP) blood pressure, lipid profile, total cholesterol (TC), HDL, LDL, triglycerides (TG) and glucose. The Framingham score was used to estimate the 10-year risk for coronary heart disease. Results: Except for $L D L$ and TG values, most subjects were within the reference limits for the analyzed variables. Thirteen subjects (92.7\%) were below the range of 10\% for the 10-year risk for coronary heart disease, while only one subject (7.3\%) showed a moderate risk. There was a significant correlation between training time and the SBP variable. Conclusion: We suggest conducting randomized trials to assess the same outcomes of this study.
\end{abstract}

Keywords: exercise, coronary disease, HIV/AIDS.

\section{RESUMEN}

Introducción: La terapia antirretroviral de gran actividad (HAART) se introdujo en Brasil en 1996, como parte de la política nacional de acceso gratuito a los servicios de salud y medicamentos. Desafortunadamente, su uso continuado se ha asociado con cambios en la distribución de la grasa corporal y los cambios metabólicos que pueden aumentar la morbilidad y la mortalidad en esta población. El entrenamiento físico se ha estudiado como una estrategia de intervención no farmacológica eficaz para mejorar los parámetros de la condición física relacionados con la salud y reducir al mínimo los efectos adversos de la infección por VIH y/o el uso prolongado de la terapia HAART, sin embargo, existen pocos estudios sobre entrenamiento físico, el síndrome de lipodistrofia y cardiología. Objetivo: Evaluar el riesgo de cardiopatía isquémica en pacientes con VIH/SIDA que reciben HAART que practican entrenamiento concurrente con series simples. Métodos: Catorce sujetos se evaluaron utilizando la circunferencia de 
cintura, presión arterial sistólica (PAS) y diastólica (PAD), colesterol total (CT), HDL, LDL, triglicéridos (TG) y la glucosa. Para estimar el riesgo coronario en 10 años se utilizó el score de Framingham. Resultados: La mayoría de los sujetos estaban dentro de los límites de referencia de las variables analizadas, a excepción de LDL y TG. Trece sujetos (92,7\%) estaban por debajo del riesgo coronario del 10\% en 10 años, y sólo uno (7,3\%) tenía riesgo moderado. Hubo una correlación significativa entre la duración del entrenamiento y la PAS. Conclusión: Se sugiere llevar a cabo ensayos clínicos aleatorios para evaluar los mismos resultados de este estudio

Palabras clave: ejercicio, coronariopatía, VIH/SIDA.

\section{INTRODUÇÃO}

A terapia antirretroviral combinada (TARV) foi introduzida no Brasil em 1996 como parte da política nacional de livre acesso aos serviços de saúde e medicamentos, sendo disponibilizada para os pacientes HIV/AIDS com indicação de tratamento'. Dados mundiais revelam que, no final de 2012, cerca de 9,7 milhões de pessoas estavam utilizando a TARV; um aumento de 1,6 milhões em relação ao ano anterior ${ }^{2}$. A TARV alterou a história natural da AIDS, tornando-a uma entidade crônica, pela supressão sustentada da carga viral e, como consequência, a reconstituição imunológica.

Infelizmente, o seu uso continuado tem sido associado às alterações na distribuição da gordura corporal e mudanças metabólicas, como resistência à insulina, hiperlipidemia, alterações ósseas e renais e doenças cardiovasculares, as quais podem aumentar a morbimortalidade nestes indivíduos ${ }^{3}$.

Salienta-se que a infecção pelo HIV causa anormalidades que incluem níveis elevados de triglicerídeos (TG), oscilações do colesterol total (CT) e colesterol de alta densidade (HDL), com graus de variação de acordo com a supressão imunológica ${ }^{4,5}$. A dislipidemia foi descrita em pessoas infectadas pelo HIV desde 1989, ou seja, antes do advento da TARV ${ }^{4}$. A associação dos efeitos adversos provenientes do uso contínuo da TARV e da própria infecção pelo HIV potencializa o desenvolvimento de eventos cardiovasculares ${ }^{6,7,8}$.

O treinamento físico tem sido estudado como uma estratégia de intervenção não farmacológica efetiva para melhorar os parâmetros de aptidão física relacionados à saúde e minimizar os efeitos indesejáveis da infecção pelo HIV e do uso prolongado da TARV9,10, porém, são escassos os estudos com treinamento físico, síndrome lipodistrófica e cardiologia. O objetivo do estudo foi avaliar o risco de doença isquêmica cardíaca em sujeitos HIV/AIDS em uso de TARV, praticantes de treinamento concorrente (associação dos componentes aeróbio e de força na mesma sessão de treinamento) com séries simples (uma série por exercício de força).

\section{MÉTODOS}

O estudo caracterizou-se como transversal, composto por amostragem consecutiva de 14 sujeitos (12 homens e duas mulheres) portadores de HIV/AIDS que utilizavam TARV, no mínimo, por três meses, e que não estavam em uso de medicamentos hipolipemiantes e acompanhamento nutricional; integrantes do Projeto Pró-Vida (UFRGS): Programa de Exercícios Físicos para Pacientes HIV. O projeto foi aprovado pelo Comitê de Ética em Pesquisa do Hospital de Clínicas de Porto Alegre (HCPA), RS, Brasil.

O programa de treinamento do Pró-Vida consiste em exercício aeróbio (em cicloergômetro) de intensidade moderada e força de resistência (em aparelhos e pesos livres). A intensidade individual do treinamento aeróbio é monitorada pela frequência cardíaca, através de cardiotacômetro da marca Polar, modelo FS1, correspondendo inicialmente a $60 \%$ do $\mathrm{VO}_{2}$ pico e mantido a $70 \%$ durante o treina- mento. No treinamento de força, a partir da carga obtida no teste de 15 repetições máximas (15 RM), as sessões são desenvolvidas em séries simples de 15 repetições para os exercícios voador, roldana alta, pressão de pernas, roscas bíceps e tríceps. A execução de 15 RM corresponde à intensidade moderada, ou seja, 80 a 85\% de 1 $\mathrm{RM}^{11}$. O abdome é treinado a uma intensidade de $50 \%$ do número de máximo de repetições obtido no teste de flexão de tronco ${ }^{12}$. A frequência é de três sessões semanais e o tempo total de cada sessão tem a duração de aproximadamente 40 minutos.

O período de permanência de cada paciente no programa foi estabelecido de acordo com a data da sua adesão ao projeto até a data da coleta dos dados.

\section{A coleta dos dados foi realizada nas seguintes etapas:}

a. Entrevista estruturada para a coleta dos dados clínicos.

b. Circunferência da Cintura: foi mensurada no plano horizontal, na metade da distância entre a crista ilíaca e o rebordo costal inferior ao final de uma expiração normal, sem compressão da pele ${ }^{13}$ utilizando-se uma fita antropométrica inextensível da marca Sanny ${ }^{\circledR}$. O ponto de corte adotado para homens foi $<102 \mathrm{~cm}$ e para mulheres $<88 \mathrm{~cm}^{14}$

c. Pressão Arterial Sistólica (PAS) e Pressão Arterial Diastólica (PAD) de repouso: mediu-se com aparelho da marca BIC no mesmo dia das coletas de acordo com as normas da VI Diretrizes Brasileiras de Hipertensão ${ }^{15}$.

d. Punção venosa: as coletas de sangue foram realizadas com sujeitos em jejum de 12 horas, sendo coletados $16 \mathrm{ml}$ destinados às análises de carga viral, contagem de Linfócitos T CD4+/T CD8+ e as dosagens bioquímicas. Respeitou-se, para cada coleta, um período de 48h após a última sessão de treino para que não houvesse nenhuma interferência do efeito agudo do exercício sobre as variáveis estudadas.

e. Mensuração da carga viral: utilizou-se a técnica Versant HIV-1 RNA 3.0 Assay/ bDNA com analisador Quantiplex System 340 bDNA. As contagens de linfócitos TCD4+ e TCD8+ foram realizadas por citometria de fluxo através do equipamento BD Facs-Scalibur 2001.

f. Dosagens de CT, HDL, LDL, TG e Glicemia (GLI): foram realizadas por colorimetria na automação bioquímica Cobas c111 da marca Roche. Utilizou-se como valores de referência para perfil lipídico: CT $<200$ $\mathrm{mg} / \mathrm{dL} ; \mathrm{TG}<150 \mathrm{mg} / \mathrm{dl} ; \mathrm{HDL}$ Homem > 40 e Mulher > 50mg/dl ${ }^{14}$, $\mathrm{LDL}<100 \mathrm{mg} / \mathrm{dL}^{16}$ e para a GLI: $<100 \mathrm{mg} / \mathrm{dl}^{17}$.

Para o cálculo da estimativa de risco coronariano em 10 anos utilizou-se o Escore de Framingham (FRM) ${ }^{18,19}$. Foram estabelecidos como ponto de corte valores menores que 10\% como baixo risco, valores de $10-20 \%$ como risco moderado e valores superiores a $20 \%$ como risco elevado ${ }^{18,19}$.

Os dados estão expressos em mediana, amplitude e percentual. Para avaliar a associação entre o período de permanência no programa de treinamento e os desfechos foi utilizada a correlação de Spearman $(p<0,05)$. O programa estatístico foi o SPSS 16.0. 


\section{RESULTADOS}

A tabela 1 apresenta as características clínicas dos sujeitos.

A tabela 2 descreve a mediana e a amplitude das variáveis hemodinâmicas (PAS, PAD), bioquímicas (TG, CT, HDL, LDL, GLI) e antropométrica dos sujeitos com a sua classificação de acordo com os valores de referência.

Pelo cálculo do Escore de Framingham observou-se que 13 sujeitos $(92,7 \%)$ ficaram abaixo dos $10 \%$ de risco coronariano em 10 anos, e apenas um (7,3\%) estava em risco moderado (12\% de risco coronariano). Ressalta-se que o único sujeito caracterizado como de risco moderado era também o único idoso (67 anos) da amostra (figura 1).

Não foi observada associação significativa entre o período de permanência no programa de treinamento e a estimativa de risco coronariano em 10 anos (tabela 3).

Na tabela 3 constam os valores das correlações das variáveis com o tempo de treinamento concorrente com séries simples. Houve associação significativa entre o tempo de treinamento e a variável PAS.

Tabela 1. Características clínicas.

\begin{tabular}{c|c}
\hline Variáveis & (n=14) \\
\hline Idade (anos) & $44,2(28-67)$ \\
\hline Sexo & $12(85,7 \%)$ \\
\hline Masculino & $2(14,3 \%)$ \\
\hline Feminino & $9(64,2 \%)$ \\
\hline Esquema Terapêutico & $1(7,1 \%)$ \\
\hline 1IP+ 2ITRN + 1IF & $2(14,2 \%)$ \\
\hline 2ITRN + 1ITRNN & $2(14,2 \%)$ \\
\hline 2 ITRN + 2 ITRN & $141,8(18-240)$ \\
\hline Período de uso da TARV (meses) & $744(134-1941)$ \\
\hline T CD4+ (células/ml) & $12(85,7 \%)$ \\
\hline Carga viral (cópias/ml) & $1(7,1 \%)$ \\
\hline$<50$ & $1(7,1 \%)$ \\
\hline Período de permanência no programa (meses) & $21,5(8-48)$ \\
\hline ITRN: Inibidor de Transcriptase Reversa análogo de Nucleosídeo; ITRNN: Inibidor de Transcriptase Reversa não-
\end{tabular}

-análogo de Nucleosídeo; IP: Inibidor de Protease; IF: Inibidor de Fusão.

Tabela 2. Variáveis sanguíneas, bioquímicas e antropométricas.

\begin{tabular}{c|c|c|c}
\hline Variáveis & $\begin{array}{c}\text { Resultados } \\
\text { mediana e } \\
\text { amplitude }\end{array}$ & $\begin{array}{c}\text { Número de sujeitos } \\
\text { dentro dos valores de } \\
\text { referência }\end{array}$ & $\begin{array}{c}\text { Número de sujeitos } \\
\text { fora dos valores de } \\
\text { referência }\end{array}$ \\
\hline PAS & $110(100-140)$ & 13 & $1^{*}$ \\
\hline PAD & $70(70-90)$ & 13 & $1^{*}$ \\
\hline TG & $192,7(53-518)$ & 5 & 3 \\
\hline CT & $186,07(133-269)$ & 11 & 5 \\
\hline HDL & $46,3(24-63)$ & 9 & 7 \\
\hline LDL & $101,5(46-145)$ & 7 & 2 \\
\hline$G L I$ & $84(63-106)$ & 12 & 0 \\
\hline CC & $82(71-102)$ & 14 & \\
\hline
\end{tabular}

PAS: Pressão arterial sistólica (mmHg); PAD: Pressão arterial diastólica (mmHg); TG: Triglicerídeos (mg/dL); CT: colesterol total (mg/dL); HDL (mg/dL); LDL (mg/dL); Glicemia (mg/dL); CC: circunferência da cintura (centímetros); (*): Hipertensão arterial estágio 1.

\section{DISCUSSÃO}

O escore de Framingham é amplamente aceito e recomendado pelo The Adult Treatment Panel III (ATP III) do National Cholesterol Education Program para estimar o risco absoluto de eventos cardiovasculares. O escore pode ser utilizado para identificar e monitorar efeitos de diferentes tipos de tratamentos, inclusive do treinamento físico.

Triant et al., ${ }^{20}$ em um estudo de coorte, evidenciaram que os portadores de HIV/AIDS apresentam uma prevalência maior de hipertensão arterial sistêmica quando comparados aos não-portadores (21,2\% vs. $15,9 \%)$. No presente estudo evidenciou-se que a maioria dos sujeitos era normotensa $(93,7 \%)$ e somente um $(7,3 \%)$ individuo foi classificado

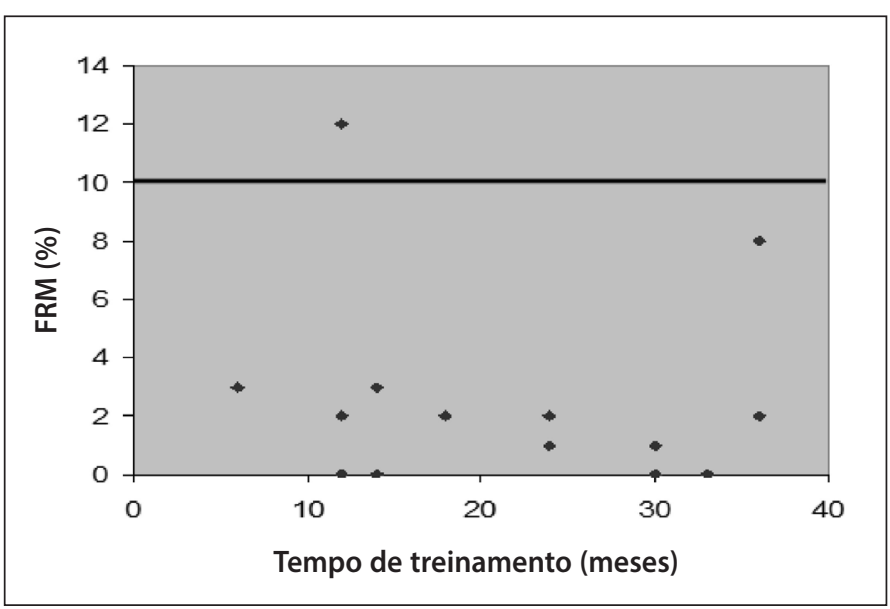

Figura 1. Escore de Framingham (\%) em função do tempo de treinamento (linha divisória de baixo risco).

Tabela 3. Correlação das variáveis estudadas com o tempo de treinamento.

\begin{tabular}{c|c|c}
\hline Variáveis & Correlação & $\mathbf{p}$ \\
\hline TG & 0,410 & 0,073 \\
\hline CT & 0,350 & 0,110 \\
\hline$H D L$ & $-0,291$ & 0,156 \\
\hline$L D L$ & 0,416 & 0,069 \\
\hline$G L$ & 0,016 & 0,479 \\
\hline CC & 0,035 & 0,453 \\
\hline PAS & $-0,505$ & 0,033 \\
\hline PAD & $-0,311$ & 0,140 \\
\hline FRA & $-0,063$ & 0,416
\end{tabular}

TG. Triglicerídeos (mg/dL): CT. colesterol total (mg/dL): HDL (mg/dL): LDL (mg/dL): Glicemia (mg/dL): FRM: Escore de Framingham (\%); CC: circunferência da cintura; PAS: Pressão arterial sistólica (mmHg); PAD: Pressão arterial diastólica $(\mathrm{mmHg})$

como hipertenso estágio um. Esse dado associado à permanência mínima de oito meses no programa de treinamento, corrobora com o pressuposto de que, mesmo em portadores de HIV/AIDS que fazem uso da TARV, o treinamento físico atua na regulação da pressão arterial21, principalmente na PAS.

Os sujeitos mantiveram-se dentro dos valores de referência para CT e HDL, porém, nove estavam acima dos valores para triglicerídeos (<150mg/dL), com mediana de 192,7 mg/dL. Para os valores de LDL, sete (50\%) sujeitos estavam dentro dos limites de normalidade $(<100 \mathrm{mg} / \mathrm{dL})$. O treinamento físico estaria minimizando os efeitos adversos da TARV sobre o perfil lipídico, mas não o suficiente para manter todas as variáveis dentro dos valores de referência. Em um ensaio clínico randomizado, Terry et al. ${ }^{22}$ estudaram 30 portadores de HIV/AIDS em uso de TARV, com dislipidemia e lipodistrofia, que participaram de um programa de treinamento aeróbio com controle nutricional e, após 12 semanas, foi verificado que não houve nenhuma alteração benéfica significativa nos níveis plasmáticos dos lipídeos.

Outros estudos também avaliaram os níveis de colesterol total, LDL e triglicerídeos dos portadores de HIV/AIDS em uso de TARV. Yarasheski et al. ${ }^{23}$ utilizaram um protocolo de 16 semanas com treinamento de força com séries múltiplas e identificaram redução significativa nos níveis de triglicerídeos e manutenção nos níveis de HDL e LDL colesterol. Engelson et al. ${ }^{24}$ avaliaram o treinamento concorrente com séries múltiplas associado a uma restrição calórica em 18 mulheres obesas HIV/AIDS em uso de TARV. Elas foram acompanhadas durante 12 semanas e apresentaram diminuição de massa corporal total e gordura corporal, mas não houve alteração significativa no perfil lipídico.

O uso da TARV está associado ao desenvolvimento de resistência à insulina e, posteriormente, hiperglicemia 25,26 , porém, nesse estudo, obteve-se uma mediana dentro dos valores ideais para GLI (84 mg/dL), 
sendo que nenhum sujeito foi identificado com glicemia alterada, corroborando que, com o treinamento físico, há uma melhoria no metabolismo da glicose, aumentando a sensibilidade à insulina ${ }^{27}$.

$\mathrm{Na}$ circunferência da cintura, todos sujeitos situaram-se dentro dos valores de referência e não houve associação significativa com o tempo de treinamento. Estudo conduzido por Kroll et al. ${ }^{28}$, com avaliação da circunferência da cintura em portadores de HIV/AIDS com e sem TARV, evidenciou que o valor em pacientes sem TARV foi inferior aqueles com esquema terapêutico com inibidor de Transcriptase Reversa Análogo de Nucleosídeo e Inibidor de Protease (87,87 $\mathrm{cm}$ versus 89,11 e 90,50 cm). Jaime et al. ${ }^{29}$, identificaram forte associação da circunferência da cintura com o sexo $(p=0,001)$, sendo a prevalência nas mulheres maior (32,7\%) do que nos homens (6,4\%). Mendes et al. ${ }^{30}$, no estudo com treinamento concorrente com séries múltiplas, evidenciou uma redução significativa na circunferência da cintura após 24 semanas de intervenção, mesmo na presença de lipodistrofia. No presente estudo, os valores da circunferência abdominal situaram-se de acordo com os valores de referência em ambos os sexos. Reitera-se a importância do seu monitoramento, pois ela permite identificar portadores de obesidade visceral e representa um marcador de risco para alterações metabólicas, independentemente do Indice de Massa Corporal (IMC) ${ }^{14}$

Há escassez de estudos que avaliem os efeitos do treinamento físico com a estimativa de risco coronariano de portadores de HIV/AIDS em uso de TARV. Um dos poucos trabalhos, foi o de Lima et. al. ${ }^{31}$, que analisaram um programa de prevenção composto por dieta controlada, treinamento físico e abandono de tabagismo na estimativa de risco coronariano em
10 anos de 87 pacientes. Após seis meses de implantação do programa foi observada a redução significativa de $92 \%$ para $27,6 \%$ nos pacientes com estimativa elevada, no entanto, não há especificações detalhadas sobre o tipo de treinamento físico abordado.

Nesse estudo não houve nenhum sujeito com estimativa de risco coronariano elevada. Observa-se que, de acordo com o escore de Framingham, o risco coronariano em 10 anos foi baixo para a expressiva maioria dos indivíduos $(92,7 \%)$, sendo que apenas um $(7,3 \%)$ obteve risco considerado moderado (12\%).

\section{CONCLUSÃO}

A maioria dos sujeitos situou-se de acordo com os valores de referência nas variáveis pressão arterial sistólica (PAS) e diastólica (PAD), colesterol total (CT), colesterol de alta densidade (HDL), glicemia (GLI) e circunferência da cintura (CC). Na variável pressão arterial sistólica (PAS) houve associação significativa com o tempo de treinamento. No risco coronariano em 10 anos, 13 sujeitos ficaram abaixo dos 10\%. Sugere-se a realização de ensaios clínicos randomizados para avaliar os desfechos deste estudo.

\section{AGRADECIMENTOS}

Os autores agradecem ao CNPq (Conselho Nacional de Desenvolvimento Tecnológico) pelo suporte financeiro.

Todos os autores declararam não haver qualquer potencial conflito de interesses referente a este artigo.

\section{REFERÊNCIAS}

1. Levi GC, Vitória MAA. Fighting against AIDS: the Brazilian experience. AIDS. 2002;16:2373-83.

2. UNAIDS (JOINT UNITED PROGRAMME ON HIV/AIDS). TREATMENT 2015 Geneva, UNAIDS 2012. Retrieved from: http://www.unaids.org/en/resource/publications/2013/name,81489,en.asp.

3. Villaroya F, Domingo P, Giralt M. Drug-inducec lipotoxicity: lipodystrophy associated with HIV-1 infection and antiretroviral therapy. Biochim. Biophys. Acta. 2010;1801(3):392-9.

4. Anastos K, Lu D, Shi, Q, Tien P, Kaplan C, Hessol NA, et al. Association of Serum Lipid Levels With HIV Serostatus, Specific Antiretroviral Agents, and Treatment Regimens. J Acquir Immune Defic Syndr. 2007:45(1):34-42

5. Kotler DP. HIV and antiretroviral therapy: lipid abnormalities and associated cardiovascular risk in HIVinfected patients. J Acquir Immune Defic Syndr.. 2008;49:79-85.

6. Zareba KM, Miller TL, Lipshultz SE. Cardiovascular disease and toxicities related to HIV infection and its therapies. Expert Opin Drug Saf. 2005;4:1017-25.

7. Van Leuven SI, Sankatsing RR, Vermeulen JN, Kastelein JJ, Reiss P,Stroes ES. Atherosclerotic vascular disease in HIV: it is not just antiretroviral therapy that hurts the heart. Curr Opin HIV AIDS. 2007;2(4):324-31.

8. Hsue PY, Hunt PW, Schnell A, Kalapus SC, Hoh R, Ganz P, et al. Role of viral replication, antiretroviral therapy, and immunodeficiency in HIV-associated atherosclerosis. AIDS. 2009;23(9):1059-67.

9. Ciccolo JT, Jowers EM, Bartholomew JB. The Benefits of Exercise Training for Quality of Life in HIV/AIDS in the Post-HAART Era. Sports Med. 2004;34(8):487-99.

10. Lazzarotto AR, Deresz LF, Sprinz E. HIV/AIDS e treinamento concorrente: a revisão sistemática; HIV/Aids and Concurrent Training: Systematic Review. Rev Bras Med Esporte. 2010;16(2):149-54

11. Kraemer WJ, Bush JA. Diretrizes do ACSM para os testes de esforço e sua prescrição: Fatores que afetam as respostas neuromusculares agudas ao exercício de resistência. 4th ed. Rio de Janeiro: Guanabara Koogan. 2003.

12. Fleck SJ, Kraemer WJ. Fundamentos do treinamento de força muscular. $2^{\circ}$ ed. Porto Alegre: Artmed Editora; 2002.

13. Carr DB, Utzschneider KM, Hull RL, Kodama K, Retzlaff BM, Brunzell JD, et al. Intra-abdominal fat is a major determinant of the National Cholesterol Education Program Adult Treatment Panel III criteria for the metabolic syndrome. Diabetes. 2004; 53(8):2087-94.

14. National Cholesterol Education Program. Third Report of the National Cholesterol Education Program (NCEP) Expert Panel on Detection, Evaluation, and Treatment of High Blood Cholesterol in Adults (Adult Treatment Panel III) final report. Circulation. 2002;106:3143-21.

15. Brandão AA, Magalhães MEC, Ávila A, Tavares A, Machado CA, Campana EMG, et al. VI Diretrizes Brasileiras de Hipertensão. Arq Bras Cardiol. 2010;95(1) supl 1: 1- 51.

16. Grundy SM, Cleeman JI, Bairey Merz CN, Jr Brewer HB, Clark LT, Hunninghake DB, et al. Implications of Recent Clinical Trials for the National Cholesterol Education Program Adult Treatment Panel III Guidelines. Circulation. 2004;110(2):227-39.
17. American Diabetes Association. Diagnosis and classification of diabetes mellitus. Diabetes Care. 2012;35:S64-71.

18. Levy RI. The Framingham Study: The Epidemiology of Atherosclerotic Disease. JAMA. 1981;245(5):512

19. D'Agostino Sr RB, Grundy S, Sullivan LM, Wilson P. Validation of the Framingham coronary heart disease prediction scores:results of a multiple ethnic groups investigation. JAMA. 2001;286(2):180-7.

20. Triant VA, Lee H, Hadigan C, Grinspoon SK. Increased acute myocardial infarction rates and cardiovascular risk factors among patients with human immunodeficiency virus disease. J Clin Endocrinol Metab. 2007;92(7):2506-12.

21. Fagard RH, Cornelissen VA. Effect of exercise on blood pressure control in hypertensive patients. Eur J Cardiovasc Prev Rehabil. 2007;14(1):12-7

22. Terry L, Sprinz E, Stein R, Medeiros NB, Oliveira J, Ribeiro JP. Exercise training in HIV-1-infected individuals with dyslipidemia and lipodystrophy. Med Sci Sports Exerc.. 2006;38(3):411-7.

23. Yarasheski KE, Tebas P, Stanerson B, Claxton S, Marin D, Bae K, et al. Resistance exercise training reduces hypertriglyceridemia in HIV-infected men treated with antiviral therapy. J Appl Physiol. 2001;90(1):133-8

24. Engelson ES, Agin D, Kenya S, Werber-Zion G, Luty B, Albu JB, et al. Body composition and metabolic effects of a diet and exercise weight loss regimen on obese, HIV-infected women. Metabolism. 2006;55(10):1327-36.

25. Brown TT, Li X, Cole SR, Kingsley LA, Palella FJ, Riddler SA, et al. Cumulative exposure to nucleoside analogue reverse transcriptase inhibitor is associated with insulin resistance markers in the Multicenter AIDS Cohort Study. AIDS. 2005;19(13):1375-83.

26. Samaras K, Gan SK, Peake PW, Carr A, Campbell LV. Proinflamatory markers, insulin sensitivity and cardiometabolic risk factors in treated HIV infection. Obesity. 2009;17(1):53-9.

27. Lindegaard B, Hansen T, Hvid T, Van Hall G, Plomgaard P, Ditlevsen S, et al. The effect of strength and endurance training on insulin sensitivity and fat distribution in human immunodeficiency virus-infected patients with lipodystrophy. J Clin Endocrinol Metab. 2008;93(10):3860-9.

28. Kroll AF, Sprinz E, Leal SC, Labrêa MG, Setúbal S. Prevalence of obesity and cardiovascular risk in patients with HIV/AIDS in Porto Alegre, Brazil. Arq Bras Endocrinol Metab. 2012;56(2):137-41.

29. Jaime PC, Florindo AA, Latorre MRDO, Brasil BG, Santos ECM, Segurado AAC. Prevalência de sobrepeso e obesidade abdominal em indivíduos portadores de HIV/AIDS, em uso de terapia anti-retroviral de alta potência. Rev Bras Epidemiol. 2004;7(1):65-72.

30. Mendes EL, Andaki ACR, Amorim PRS, Natali AJ, Brito CJ, Paula SO. Treinamento físico para indivíduos HIV positivo submetidos à HAART: efeitos sobre parâmetros antropométricos e funcionais. Rev Bras Med Esporte. 2013;19(1):16-21.

31. Lima EM, Gualandro DM, Yu PC, Giuliano IDEC, Marques AC, Calderaro D, et al. Cardiovascular prevention in HIV patients: Results from a successful intervention program. Atherosclerosis. 2009;204(1):229-32. 